\title{
Estimating the effect of nasal steroid treatment on repeat polypectomies: survival time analysis using the General Practice Research Database*
}

\author{
Yogesh Suresh Punekar ${ }^{1}$, Azhar Ahmad ${ }^{1}$, Hesham A. Saleh² \\ ${ }^{1}$ Schering-Plough Ltd., Hertfordshire, United Kindom \\ ${ }^{2}$ Charing Cross and Royal Brompton Hospitals, Honorary Senior Lecturer, Imperial College of Medicine, \\ London, United Kingdom
}

\begin{abstract}
SUMMARY Background: Intranasal steroids are effective in preventing or delaying recurrence of nasal polyps. However, their effectiveness in delaying a need for repeat polypectomy in clinical practice is unknown.

Objectives: To compare time to a repeat polypectomy between post-polypectomy intranasal steroid users and non-users.

Methodology/Principle: Our cohort consisted of patients in GPRD who had undergone at least one nasal polypectomy procedure in or after the year 2000. These patients were followed for up to 4 years and the time to next polypectomy was estimated. Cox's proportional hazards regression was used to estimate the effect of post polypectomy intranasal steroid treatment on time to the next polypectomy after controlling for other respiratory conditions and their treatment.

Results: The cohort consisted of 1,675 patients with a mean age of 59 years and 68\% males. Of these, 576 patients were post-polypectomy steroid users and 1,099 patients were steroid non-users. The median time to repeat polypectomy was 812 days among the steroid users and 736 days among steroid non-users. Significantly less proportion of intranasal steroid users experienced a repeat polypectomy compared to steroid non-users. This difference was consistent among subgroups of females and concomitant rhinitis treatments users. Patients with post polypectomy intranasal steroid use showed lower risk for a repeat polypectomy compared to steroid non-users. Concomitant rhinitis medication users showed a higher risk whereas other confounders were not significant.

Conclusions: Intranasal steroids were effective in delaying a repeat polypectomy. However, further research using a prospective design is necessary to quantify the benefit of ongoing steroid treatment.
\end{abstract}

Key words: Nasal polyps, polypectomy, nasal steroids, GPRD

\section{INTRODUCTION}

Nasal polyps are the most common lesions found in the nose ${ }^{(1)}$. Nasal polyps recur frequently after removal with a recurrence rate up to $40 \%$ and have been linked with allergic conditions but this has not been proven in epidemiological studies ${ }^{(2)}$. The prevalence of nasal polyps can be as high as $2.7 \%{ }^{(3)}$ and is more common in males, elderly and asthmatics ${ }^{(3)}$. Nasal polyps present significant quality of life burden especially in case of concomitant asthma and a study has reported significant improvement in quality of life for up to 10 months following medical or surgical treatment of nasal polyps ${ }^{(4)}$.

Polypectomy, which ranges from simple surgical removal of polyps to complete ethmoidectomy, is a common treatment for patients suffering from nasal polyps. Although polypectomy could be considered as a temporary cure, the polyp recurrence rates could be as high as $44 \%$ in 12 -month period requiring subsequent polypectomies ${ }^{(5)}$. A polypectomy procedure is associated with post-surgical complications. The risk of a major complication including loss of visual acuity, nasolacrimal duct damage, brain abcess, direct brain trauma and bleeding is about $0.5 \%$ whereas the risk of a minor complication could be as high as 4 percent ${ }^{(}{ }^{(}$. Nasal polyps and its surgical treatment also present a significant burden on the health care system with 11,587 hospital admissions and 8,062 bed days per year in the UK ${ }^{(7)}$. Therefore, preventing polyp recurrence becomes an important treatment goal post-polypectomy.

Intranasal steroids are often considered as treatment of choice to prevent or delay recurrence of polyps ${ }^{(5,8)}$. The British Society of Allergy and Clinical Immunology (BSACI) guidelines suggest long term maintenance treatment with fluticasone or mometasone to avoid recurrence of polyps ${ }^{(9)}$. Several randomized trials have demonstrated the beneficial impact 
of intranasal corticosteroids in delaying the relapse of polyps ${ }^{(10-12)}$. However, the long term benefit of intranasal steroid treatment in actual clinical practice is yet to be demonstrated.

The objective of this study was to compare time to a repeat polypectomy between patients treated with and without intranasal steroids post polypectomy.

\section{MATERIALS AND METHODS}

\section{Patient cohort selection}

We constructed a cohort of patients post nasal polypectomy using the UK General Practice Research Database (GPRD). The GPRD is one of the largest healthcare databases in the world extracting data from 488 practices in England and Wales, comprising 3.69 million active individuals who contribute 33.12 million person-years of data. The records from this database have been validated in several previous studies ${ }^{(13-17)}$. The protocol for this study was approved by the GPRD Ethics Committee.

Our cohort consisted of patients who had undergone at least one nasal polypectomy procedure in or after the year 2000 . The incidence of a polypectomy procedure was identified using relevant: 1) Oxford Medical Information System (OXMIS) codes, which were derived from the International Classification of Disease (version 8); and 2) Read codes, which are hierarchical codes commonly used in family practices in the UK. Patients with concomitant diagnosis of cystic fibrosis, nasal infection, rhinitis medicamentosa and latent or active pulmonary tuberculosis were excluded. Patients were also excluded if they had a history of more than three polypectomies in their records. The recorded date of polypectomy event was considered as index polypectomy date (IPD) and was selected randomly for patients with multiple polypectomy events. If the patient was suffering from a nasal infection at the time of selected IPD, a different IPD was selected.

\section{Treatment information}

The relevant treatment information for patients in this cohort was extracted. This included data on nasal corticosteroid use one month prior and 6 months following IPD. Patients were considered as steroid users if they had at least one prescription of nasal steroid within 3 months following IPD and had at least three steroid prescriptions in the first 6 months. Information on patient demographic characteristics available in GPRD such as age, gender and practice was also extracted. Steroid use 1 month before IPD was used as a confounding variable. Other potential confounders such as asthma, sinusitis and allergic rhinitis as well as chronic conditions such as diabetes, heart failure and COPD were identified. Treatment information on other medications which may interfere with polyps such as leukotriene receptor antagonists, antihistamines, macrolide antibiotics, aspirin, beta-blockers, NSAIDs, paracetamol and $\mathrm{H}_{2}$ antagonists as well as inhaled and systemic corticosteroids was collected.

\section{Patient follow-up}

The outcome of interest was time to the next polypectomy event following IPD. Patients were followed for a maximum period of 4 years after the IPD. For patients who did not experience a polypectomy event after IPD, their last recorded follow-up date was used. Repeat polypectomy procedures occurring within a year of the IPD were excluded as it was difficult to distinguish steroid use as prophylaxis or treatment.

\section{Statistical analysis}

Cox's proportional hazards regression was used to estimate the effect of post IPD intranasal steroid treatment on time to the next polypectomy event. The Cox's model was initially tested in a univariate analysis and then in a multivariate analysis controlling for the potential confounders. All data extraction and statistical analyses were conducted using STATA v10.0 ${ }^{(18)}$.

\section{RESULTS}

\section{Overall characteristics of cohort}

Our cohort consisted of 1,680 patients who underwent at least one polypectomy in or after the year 2000. Of these patients, 5 were suffering from cystic fibrosis and were excluded from further analysis resulting in a final cohort of 1,675 individuals. The mean age of the cohort was 59 years $(\mathrm{SD}=15.7)$ and $68 \%$ were males. No more than $1 \%(n=17)$ of patients were from a single practice and $85 \%$ of patients were registered and were active as of $31^{\text {st }}$ March 2008. All patients in the cohort were deemed to have records acceptable by GPRD quality standards.

There was no significant difference between patients using intranasal steroids post IPD and patients not using intranasal steroids post IPD on any of the demographic variables.

The medication use 1 month prior and 3 months post IPD is displayed in Table 1. More patients who used post IPD intranasal steroids were suffering from other respiratory conditions such as asthma, sinusitis or allergic rhinitis, and a significantly higher proportion was using allergy medications and inhaled or topical steroids at the time of IPD compared to patients with no post IPD intranasal steroid use. The 2 groups

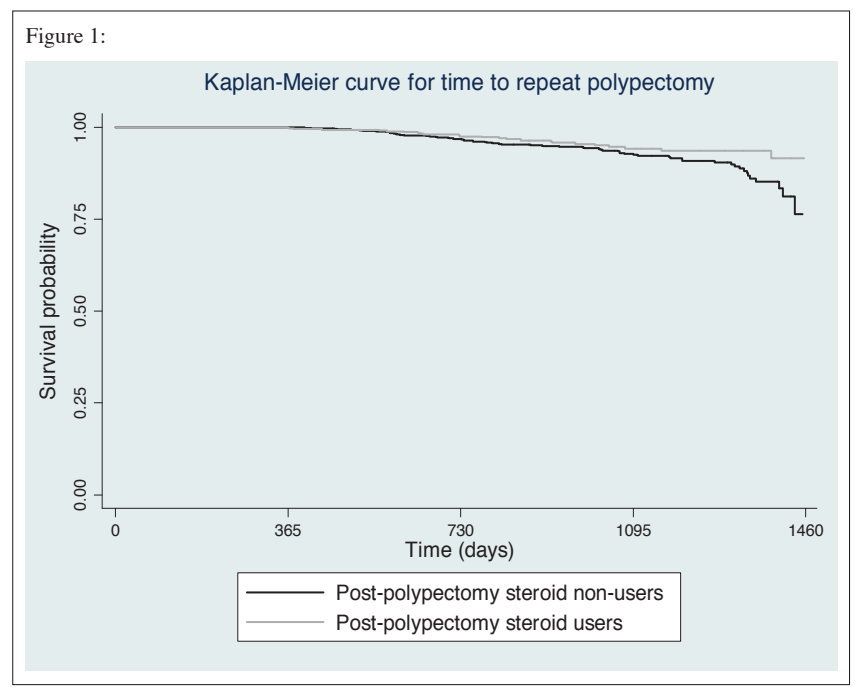




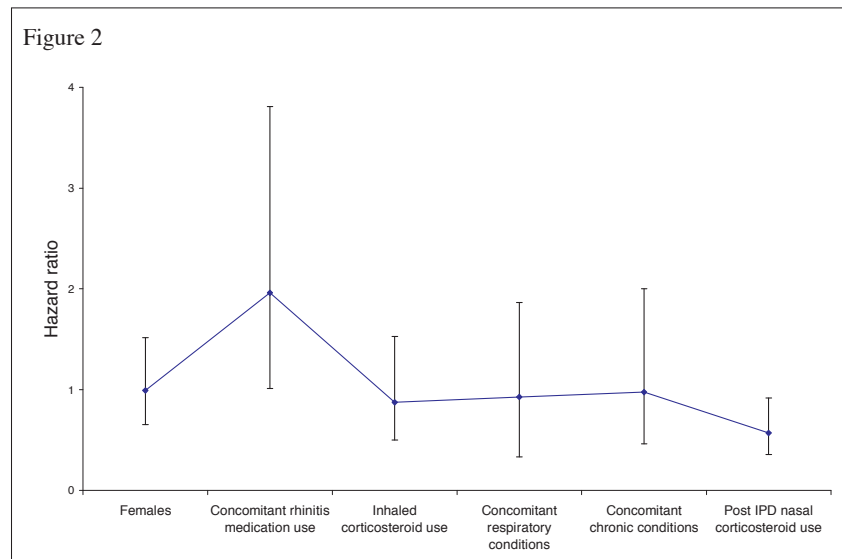

however were comparable on other chronic conditions such as diabetes, heart disease and COPD.

Time to repeat polypectomy event

The Kaplan-Meier curve displaying the time to a repeat polypectomy event among patients with and without post IPD intranasal steroid use is displayed in Figure 1. The median time to repeat polypectomy was 812 days (Inter Quartile Range (IQR): 574-1030) among post-polypectomy steroid users and 736 days (IQR: 544-1045) among steroid non-users. Significantly less proportion of patients using an intranasal steroid post IPD experienced a repeat polypectomy event in 4 years following IPD (4.1\%) compared to intranasal steroid non-users $(7.8 \%)(\mathrm{p}=0.008)$. Post IPD intranasal steroid users also showed significant reductions in repeat polypectomies among the subgroups of females $(2.3 \%$ vs $9.1 \%$; $p=0.006)$ and other concomitant rhinitis treatments users at IPD (3.9\% vs $19.1 \% ; p=0.036$ ). In contrast, the proportions of patients undergoing repeat polypectomies were comparable between post IPD intranasal steroid users and non-users among subgroups of concomitant inhaled steroid users, concomitant sufferers of a respiratory condition and sufferers of chronic conditions such as diabetes or heart disease.

Figure 2 displays the multivariate Cox proportional hazards model and the effect of confounding variables on the event of a repeat polypectomy. Post IPD intranasal steroid use was the most significant variable (Hazard ratio (HR) 0.57; confidence interval $(\mathrm{CI}) 0.35-0.92 ; \mathrm{p}=0.02$ ) with patients using intranasal steroids having significantly lower risk of a repeat polypectomy. Patients using rhinitis medications such as antihistamines and leukotriene receptor antagonists at IPD had significantly higher risk of undergoing repeat polypectomy (HR: 1.96; CI $1.01-3.81 ; \mathrm{p}=0.047$ ). The other confounders such as inhaled corticosteroid use at IPD, concomitant respiratory conditions and concomitant chronic conditions did not have significant impact on reducing the time to a repeat polypectomy.

\section{DISCUSSION}

Our study compared time to a repeat polypectomy between patients treated with intranasal steroids and patients not treated with intranasal steroids post IPD. The results showed that patients treated with an intranasal steroid had longer recurrence free survival compared to steroid non-users. Our results are broadly in agreement with a randomised controlled trial which showed that patients treated with mometasone furoate experienced longer recurrence free period ${ }^{(12)}$. In this trial, the difference in median time to recurrence of polyps between the steroid user and non-user group was 50 days in favour of steroid users. In our analysis, this difference was 76 days. The trial only assessed recurrence of polyps and did not follow patients all the way up to repeat polypectomy. Due to these differing outcomes, we selected the timeframe of our analysis to be in the range of 1 to 4 years in contrast with Stjarne and others who assessed polyp recurrence up to a period of 24 weeks. Other corticosteroids such as fluticasone propionate and budesonide have also been found to have beneficial impact on polyp recurrence following a polypectomy ${ }^{(10,11,19)}$. The results of our analysis are also in agreement with the study conducted by Rowe-Jones and colleagues (11). In the study, Rowe-Jones and colleagues found that the patients treated with fluticasone propionate had significantly lower nasal polyp score until fourth year after functional endoscopic sinus surgery (FESS) but was not significant fifth year onwards. This suggested differential benefit of steroid to be maintained for about 4 years following surgery. Our results also showed this benefit to last for at least 4 years as displayed in Figure 1.

In our analysis, we selected patients who had their index polypectomy in or after the year 2000. Nasal steroids received their licences in nasal polyp treatment after the year 2000 and even though ENT surgeons have been using intranasal steroids since early nineties, its use in general practice has picked up in the last decade. This was confirmed by the post IPD treatment data which showed $21 \%$ intranasal steroid users between 1990 and 1999, and 52\% intranasal steroid users in or after the year 2000. We excluded patients who had certain concomitant conditions such as rhinitis medicamentosa, nasal infection and latent or active pulmonary tuberculosis. Rhinitis medicamentosa is secondary to decongestants abuse and is common in non-allergic rhinitis, a condition that does not respond well to nasal steroids. Nasal infection is on the other end of the spectrum of nasal inflammatory conditions and is therefore a confounding variable whereas tuberculosis although very rare can have nasal presentations or a theoretical risk of getting worse because of the nasal steroids. We also excluded patients with cystic fibrosis as these patients have neutrophilic polyps which do not respond to steroid treatment. Patients with more than three polypectomies also were excluded as having higher propensity for polyp development which could have biased the results. However, to investigate this possible bias, a separate analysis was conducted including these patients. Results showed a minor reduction in median time to repeat polypectomy in both the steroid user and the steroid non-user group with the difference remaining significant.

The current analysis focussed on repeat polypectomy event 


\begin{tabular}{|l|c|c|c|}
\hline Baseline characteristic & $\begin{array}{c}\text { Post- } \\
\text { polypectomy } \\
\text { steroid users } \\
{[\mathrm{n}=576(\%)]}\end{array}$ & $\begin{array}{c}\text { Post- } \\
\text { polypectomy } \\
\text { steroid } \\
\text { non-users } \\
{[\mathrm{n}=1,099(\%)]}\end{array}$ & $\mathrm{p}$-value \\
\hline Age (mean, years) & 61.6 & 57.0 & $\mathrm{NS}$ \\
\hline Males & $379(65.8)$ & $764(69.5)$ & $\mathrm{NS}$ \\
\hline $\begin{array}{l}\text { Currently registered in } \\
\text { the same practice }\end{array}$ & $491(85.3)$ & $934(85.0)$ & $\mathrm{NS}$ \\
\hline $\begin{array}{l}\text { Pre-polypectomy steroid } \\
\text { use }\end{array}$ & $326(56.6)$ & $251(22.8)$ & $<0.0001$ \\
\hline $\begin{array}{l}\text { Concomitant allergy } \\
\text { med use }\end{array}$ & $53(9.2)$ & $50(4.6)$ & $<0.0001$ \\
\hline Inhaled steroid use & $140(24.3)$ & $160(14.6)$ & $<0.0001$ \\
\hline $\begin{array}{l}\text { Other respiratory condi- } \\
\text { tion }\end{array}$ & $184(31.9)$ & $198(18.0)$ & $<0.0001$ \\
\hline Chronic conditions & $104(18.1)$ & $214(19.5)$ & $\mathrm{NS}$ \\
\hline
\end{tabular}

Table 1. Concomitant medication use at baseline between post IPD intranasal steroid users and non-users

beyond first year of treatment and up to 4 years. The majority of polypectomies result in some residual polyps and therefore it was difficult to attribute post surgery immediate steroid use to prophylaxis or polyp treatment. Therefore, we excluded the polypectomies within the first year post surgery to ensure prophylactic intranasal steroid use within our cohort. This may however have introduced bias. We therefore conducted a separate analysis including all repeat polypectomies. The results, although numerically different, suggested similar trend. Our analysis also was censored at the end of fourth year as 6 months of intranasal steroid treatment post-polypectomy was unlikely to have a longer lasting impact on polyp recurrence. A longer follow-up period may also have confounded the analysis further due to other respiratory conditions and their treatments.

The major strength of our analysis lies in the patient numbers and length of follow-up available in GPRD. The longitudinal data available on most patients within GPRD allowed us to control for the impact of concomitant conditions including the respiratory conditions and the associated treatments. GPRD collects data from a national sample of general practices within UK. Our results therefore are broadly generalizable to the UK population.

This study also has a number of potential limitations. The polypectomy events were exclusively identified based on read codes and OXMIS codes available in the patient records in their general practices. No additional information on the type and severity of the polyps at the time of polypectomy was available. The impact of these clinical variables on the recurrence of the polyps and the subsequent repeat polypectomy is therefore unknown. The recurrence of polyps may also be impacted by the type and nature of the surgery performed. The surgeons that are better trained surgically may perform more extensive polypectomies and are also likely to prescribe corticosteroids. This confounding effect may have biased the results in favour of corticosteroids. However, with the limited information available from GPRD, it is not possible to estimate the extent of this bias and therefore remains a major limitation of this analysis. The analysis also relies on the assumption that the mean referral time from recurrence of polyps to polypectomy was similar between intranasal steroid user group and non-user group. The numbers of patients selected from individual practices were similar in both groups and therefore we believe that any variation in referral times between individual practices will not affect the results. This assumption however could not be verified. Potential limitations of this work also lie in the limited number of demographic variables available in GPRD. The impact of socioeconomic variables such as geographic location, access to ENT services and other health system related variables could not be assessed. However, once again, the comparable proportions of patients in each group being selected from individual practice may have helped minimize any bias.

In conclusion, intranasal steroids were effective in delaying a repeat polypectomy. However, further research using a prospective design is necessary to quantify the benefit of ongoing steroid treatment.

\section{ACKNOWLEDGEMENTS}

The authors would like to acknowledge the protocol development support provided by Dr. Farhat Rasul and manuscript review support provided by Karen Durkin.

Yogesh Punekar designed the study and conducted the analysis. Azhar Ahmad and Hesham Saleh contributed to the protocol, study design and writing of the manuscript.

\section{STATEMENT OF INTEREST}

Yogesh Punekar and Azhar Ahmad are employed by Schering-Plough Ltd. Hesham Saleh has served as a speaker, a consultant and an advisory board member for ScheringPlough Ltd, and has received research funding from Schering Plough Ltd for advice on this project.

This study was funded in full by Schering-Plough Ltd, and the writing of this paper was funded in full by Schering-Plough Ltd. Writing support was provided by Suyash Enterprises and funded by Schering-Plough Ltd.

\section{REFERENCES}

1. Bernstein JM. Nasal polyps: finding the cause, determining treatment. J Respir Dis. 1997; 18: 847-856.

2. Vento S, Ertama L, Hytönen M, et al. Nasal polyposis: clinical course during 20 years. Ann Allergy Asthma Immunol. 2000; 85: 209-214.

3. Johansson L, Akerlund A, Holmberg K, et al. Prevalence of nasal polyps in adults: the Skövde population-based study. Ann Otol Rhinol Laryngol. 2003; 112: 625-629.

4. Radenne F, Lamblin C, Vandezande L, et al. Quality of life in nasal polyposis. J Allergy Clinical Immunol. 1999; 104: 79-84.

5. Bross-Soriano D, Arrieta-Gomez JR, Prado-Calleros H. Infections after endoscopic polypectomy using nasal steroids. Otolaryngol Head Neck Surg. 2004; 130: 319-322.

6. Fokkens WJ, Lund VJ, Mullol J, et al. European Position Paper on Nasal Polyps. Rhinology. 2007; 45: 1-139.

7. Department of Health (England). Hospital inpatient data - based 
on hospital episode statistics 2008-2009. URL: http://www.doh. gov.uk (accessed 21 Nov 2009)

8. Kanai N, Denburg J, Jordana M, et al. Nasal polyp inflammation. Effect of topical nasal steroid. Am J Respir Crit Care Med. 1994; 150: 1094-1100.

9. Scadding G, Durham S, Mirakian R, et al. BSACI guidelines for the management of rhinosinusitis and nasal polyposis. Clin Exp Allergy. 2007; 38: 260-275.

10. Dijkstra M, Ebbens R, Poublon R, et al. Fluticasone propionate aqueous nasal spray does not influence the recurrence rate of chronic rhinosinusitis and nasal polyps 1 year after functional endoscopic sinus surgery. Clin Exp Allergy. 2004; 34: 1395-1400.

11. Rowe-Jones J, Medcalf M, Durham S, et al. Functional endoscopic sinus surgery: 5 year follow up and results of a prospective, randomised, stratified, double-blind, placebo controlled study of postoperative fluticasone propionate aqueous nasal spray. Rhinology. 2005; 43: 2-10.

12. Stjarne $\mathrm{P}$, Olsson $\mathrm{P}$, Alenius $\mathrm{M}$. Use of mometasone furoate to prevent polyp relapse after endoscopic sinus surgery. Arch Otolaryngol Head Neck Surg. 2009 135: 296-302.

13. Hansell A, Hollowell J, Nichols T, et al. Use of the General Practice Research Database (GPRD) for respiratory epidemiology: a comparison with the 4th Morbidity Survey in General Practice (MSGP4). Thorax. 1999; 54: 413-419.

14. Nazareth I, King M, Haines A, et al. Accuracy of diagnosis of psychosis on general practice computer system. BMJ. 1993; 307: 32-34.
15. Peng M, Jick H. A Population-Based Study of the Incidence, Cause, and Severity of Anaphylaxis in the United Kingdom. Arch Int Med. 2004; 164: 317-319.

16. van Staa T-P, Abenhaim L. The quality of information recorded on a UK database of primary care records: A study of hospitalisations due to hypoglycaemia and other conditions. Pharmacoepidemiol Drug Safety. 1994; 3: 15-21.

17. Walley T, Mantgani A. The UK General Practice Research Database. Lancet. 1997; 350: 1097-1099.

18. StataCorp 1985-2009. Intercooled Stata 10.0 for Windows. College Station, TX: Stata Corporation.

19. Hartwig $\mathrm{S}$, Lindén $\mathrm{M}$, Laurent $\mathrm{C}$, et al. Budesonide nasal spray as prophylactic treatment after polypectomy (a double blind clinical trial). J Laryngol Otol. 1988; 102: 148-151.

Yogesh Punekar

Schering-Plough Ltd.

Shirepark, Welwyn Garden City

Hertfordshire, AL7 1TW

United Kingdom

Tel: +44-1707-363994

E-mail:Yogesh.punekar@spcorp.co 